\title{
Quantitative analysis of airway abnormalities in CT
}

Petersen, Jens; Lo, Pechin Chien Pau; Nielsen, Mads; Edula, Goutham; Ashraf, Haseem; Dirksen, Asger; de Bruijne, Marleen

Published in:

Medical Imaging 2010

DOI:

$10.1117 / 12.843937$

Publication date:

2010

Document version

Peer reviewed version

Citation for published version (APA):

Petersen, J., Lo, P. C. P., Nielsen, M., Edula, G., Ashraf, H., Dirksen, A., \& de Bruijne, M. (2010). Quantitative analysis of airway abnormalities in CT. In N. Karssemeijer, \& R. M. Summers (Eds.), Medical Imaging 2010: computer-aided Diagnosis [76241S] SPIE - International Society for Optical Engineering. Progress in Biomedical Optics and Imaging Vol. 11 No. 34 https://doi.org/10.1117/12.843937 


\title{
Quantitative analysis of airway abnormalities in CT
}

\author{
Jens Petersen ${ }^{a}$, Pechin Lo $^{a}$, Mads Nielsen ${ }^{a}$, Goutham Edula $^{b}$, Haseem Ashraf $^{c}$, Asger Dirksen $^{c}$ \\ and Marleen de Bruijne ${ }^{a, d}$ \\ ${ }^{a}$ Image Group, Department of Computer Science, University of Copenhagen, Denmark \\ ${ }^{b}$ Department of Respiratory \& Inflammation TA, AstraZeneca R\&D Lund, Sweden \\ ${ }^{c}$ Department of Respiratory Medicine, Gentofte Hospital, Denmark \\ ${ }^{d}$ Biomedical Imaging Group Rotterdam, Departments of Radiology \& Medical Informatics, \\ Erasmus MC, Rotterdam, The Netherlands
}

\begin{abstract}
A coupled surface graph cut algorithm for airway wall segmentation from Computed Tomography (CT) images is presented. Using cost functions that highlight both inner and outer wall borders, the method combines the search for both borders into one graph cut.

The proposed method is evaluated on 173 manually segmented images extracted from 15 different subjects and shown to give accurate results, with $37 \%$ less errors than the Full Width at Half Maximum (FWHM) algorithm and $62 \%$ less than a similar graph cut method without coupled surfaces. Common measures of airway wall thickness such as the Interior Area (IA) and Wall Area percentage (WA\%) was measured by the proposed method on a total of $723 \mathrm{CT}$ scans from a lung cancer screening study. These measures were significantly different for participants with Chronic Obstructive Pulmonary Disease (COPD) compared to asymptomatic participants. Furthermore, reproducibility was good as confirmed by repeat scans and the measures correlated well with the outcomes of pulmonary function tests, demonstrating the use of the algorithm as a COPD diagnostic tool.
\end{abstract}

Additionally, a new measure of airway wall thickness is proposed, Normalized Wall Intensity Sum (NWIS). NWIS is shown to correlate better with lung function test values and to be more reproducible than previous measures IA, WA\% and airway wall thickness at a lumen perimeter of $10 \mathrm{~mm}$ (PI10).

Keywords: Airway, COPD, graph cut, coupled surface, lung function

\section{INTRODUCTION}

The progression of Chronic Obstructive Pulmonary Disease (COPD) is associated with destruction of tissue in the human lungs, known as emphysema and thickening of the human airway walls ${ }^{1}$ along with a narrowing of the peripheral airways, which is defined as airways with an internal or so called lumen diameter of less than 2 $\mathrm{mm} .{ }^{2}$ It is therefore important for the diagnosis and monitoring of this disease to be able to accurately measure emphysema and the dimensions of the human airways and airway walls.

In order to measure the changes to the airways caused by COPD, a segmentation of the inner and outer area of the airway is usually performed. This is often done in two-dimensional cross-sections, lying perpendicular to the airway centerlines because of the difficulty in accurately segmenting in three-dimensions and across airway branchpoints. ${ }^{6-10}$ Often a region growing ${ }^{10}$ or an algorithm based on fuzzy connectivity ${ }^{8,9,11}$ is used to do an initial three-dimensional segmentation of the airways, from this the centerlines can by calculated, the branch-points avoided and the cross-sectional images computed. ${ }^{6-10}$ For a recent comparison of several different algorithms for airway extraction, see Ref. 12.

The literature contains many approaches to solve the problem of segmenting the airway wall in cross-sectional airway images. ${ }^{6-8,10,13}$ The simplest and probably the most used method is to determine the position of the wall edges via the Full Width at Half Maximum (FWHM) principle. Edges are simply defined to be at the

Further author information:(Send correspondence to J.P.)

J.P.: E-mail: phup@diku.dk, Telephone: +45 60687733

M.B.: E-mail: marleen@diku.dk, Telephone: +45 35321445 
position half-way between a consecutive minimum and maximum in intensity. Rays are cast 360 degrees around the center of the airway and the FWHM principle is applied twice to find the inner and outer border in each ray. The method has been shown to have problems when the dimensions of the measured structure are close to the size of the scanner Point Spread Function (PSF). ${ }^{13,14}$ Methods such as the phase congruency approach, ${ }^{6}$ which uses maximum phase congruency defined via multiple reconstruction kernels or the method described in Ref. 10, which uses a calibration factor representing scanner parameters, are solutions to this problem. However because all these methods use no or very simple methods, such as ellipse fitting, to combine the information in each individually sampled ray, two- and three-dimensional information is lost. This makes them vulnerable in areas where the airway borders are weakly defined or less suitable to derive information about the surface of the airway, such as curvature, smoothness etc.

Methods that rely on a cost or energy function to define the airway border position have become increasingly popular. ${ }^{7,8,11,15}$ In Ref. 8 a cost function was used to segment the surface of the airway wall and the method was verified on Plexiglas phantoms. Ref. 7 uses boundary specific cost functions defined for detecting both inner and outer borders and cost are specified so that the outer wall is very unlikely to cross the inner.

Cost function have been minimized using as in Ref. 7,8 dynamic programming or as in Ref. 11,15 graph cut methods. Graph cut or minimum path based methods are the solutions to the classical combinatorial problems of the minimum $s-t$ cut and the maximum flow problem. The first use of these algorithms in image analysis occurred in Ref. 16 and they were then later on used for airway analysis in Ref. 11, which contains the first (to our knowledge) description of how to construct the graph. The advantage compared to FWHM is that the solution can be constrained to be arbitrarily continuous with a so called smoothness constraint. A recent graph cut algorithm ${ }^{15}$ also uses constraints on inter-surface distances, enabling both inner and outer borders to be found simultaneously. By combining information of the image at the site of both borders, this coupled surface graph cut algorithm can use clues from one border to help place the other.

Two of the most often reported measures of airway narrowing and wall thickening are the Interior Area (IA) and Wall Area percentage (WA\%), ${ }^{1,5,17-21}$ which is the area of the lumen and the percentage of the total airway area which is wall respectively. These measures are often averaged over all samples in the airway tree, which makes them dependent on the quality of the airway segmentation algorithm and where in the airway tree they are sampled. Airway wall thickness at a lumen perimeter of $10 \mathrm{~mm}$ (PI10) is a new measure which tries to circumvent these problems, ${ }^{3,4}$ it is defined as the square root of the airway wall area taken at a lumen perimeter of $10 \mathrm{~mm}$ by using linear regression. Ref. 3 experiments with samples from cross-sections with an internal perimeter larger than 7.5 and $10 \mathrm{~mm}$ where as Ref. 4 uses a cutoff value of $6 \mathrm{~mm}$. The measure has been shown to correlate well with lung function even with relatively few sampling points. ${ }^{4}$ Peak Wall Attenuation (PWAt) ${ }^{5}$ is another new measure using the image intensity values instead of trying to estimate airway structure and size. This is useful because intensity is related to and maybe a more accurate measure of the size of imaged structures when these are close to the size of the PSF, because the blurring effect will reduce the contrast of the imaged structure. PWAt is defined as the mean of the maximum intensity found within the wall along rays cast 360 degrees around the center and out. It has been shown to be comparable to WA\% when it comes to correlation with lung function measures and capture independent information which is not assessed by WA\%. ${ }^{5}$

This paper presents a coupled surface graph cut algorithm for measuring airway lumen and wall area in computed tomography images of human lungs and evaluates its ability to diagnose COPD in a series of large scale tests. The novelty of our method includes a new graph construction technique, which uses soft instead of hard constraints to enforce solution smoothness and inner and outer border separation.

Boundary-specific cost functions, constructed with the use of weights of the positive and negative parts of the first and second order derivative of the image intensity function, are used to allow careful adjustment of the found solution to compensate for effects such as the size overestimation caused by the imaging system's PSF.

In addition a new intensity based measure of airway abnormalities involved in COPD called Normalized Wall Intensity Sum (NWIS), is introduced and compared with previously published measures Interior Area (IA), Wall Area percentage (WA\%), Airway wall thickness at a lumen perimeter of $10 \mathrm{~mm}$ (PI10), ${ }^{3,4}$ and Peak Wall Attenuation (PWAt). ${ }^{5}$ NWIS should be able to capture changes to the mass of the airway wall, whether these are caused by size or density changes. 


\section{METHODS}

\subsection{Airway segmentation}

The airway was segmented using an airway tree segmentation algorithm presented in Ref. 22, which incorporates trained local airway appearance models and uses the fact that an airway branch is always accompanied by an artery. By using the orientation of both structures as a basis for the region growing algorithm a method that is less sensitive to noise and yields better results than standard region growing algorithms was achieved. The airway centerlines, describing the lumen center location, generation number, branch direction and branch diameter were found from the segmented airway via a front propagation method described in Ref. 23. Difficult areas around the branch-points are naturally identified by the algorithm and were therefore avoided.

\subsection{Cross-sectional images}

Cross-sectional images were extracted perpendicular to the airway centerlines at segmented branches using a 1 mm spacing. Branches with generation numbers less than 3 were not used because of the expectation that the airway changes associated with COPD occur mainly in the smaller airways. ${ }^{2}$ Branches with generation numbers larger than 11 or a length less than $3 \mathrm{~mm}$ were discarded because they were found by visual inspection too often to be the product of erroneous airway segmentations or wrongly detected branch-points. The size $S \times S$ of the extracted cross-sectional images were determined conservatively in order to make sure they always contained all of the airway from the maximum radius of the corresponding branch $r$ as follows:

$$
S= \begin{cases}20 & \text { if } 3 r<20 \\ 3 r & \text { else }\end{cases}
$$

\subsection{Full width at half maximum}

FWHM measurements were performed by casting $2 S$ rays 360 degrees radially within the plane of the crosssection from the center and outward. Let $R$ be such a ray and $R_{x}$ be a smoothed first order derivative of that ray, calculated using central differences. The degree of smoothing was determined by the training algorithm, see Section 2.6. On each ray three intervals were used:

1. Interval to search for the first minimum - the inside minimum, $R\left(x_{i}\right)$.

2. Interval to search for the maximum - the wall maximum, $R\left(x_{m}\right)$.

3. Interval to search for the second minimum - the outside minimum, $R\left(x_{o}\right)$.

1 was defined as the interval from the start of the ray and until $R_{x}$ 's first maximum. 2 was defined as the interval from $R_{x}$ 's first maximum value to its second minimum value. 3 was defined as the interval from $R_{x}$ 's second minimum to the second maximum. Once these three intervals was determined and the corresponding $R\left(x_{i}\right)$, $R\left(x_{m}\right)$ and $R\left(x_{o}\right)$ values found, two new intervals were searched for the half value points. The first halfway value point were defined at the intensity value closest to $\left(R\left(x_{m}\right)+R\left(x_{i}\right)\right) / 2$ found on the ray within the interval: $\left[x_{i}, x_{m}\right]$. The second halfway value were found similarly from $\left(R\left(x_{m}\right)+R\left(x_{o}\right)\right) / 2$ in the interval: $\left[x_{m}, x_{o}\right]$.

\subsection{Cost functions}

The cross-sectional images were transformed into polar coordinates, so that the center of the airway, in Cartesian space, became stretched across the top of the polar image. Expanding circles around the Cartesian center became rows going downward in the polar image, as shown in Figure 1(a) and 1(b). Within this coordinate system the $\mathrm{x}$-coordinate corresponds to angles and the $\mathrm{y}$-coordinate to radial distance from the center.

Let $I_{y}$ and $I_{y y}$ be the first and second order derivatives in the y coordinate direction corresponding to the top to bottom direction within the polar coordinate system (center and outward radial direction within Cartesian space), then Ref. 7 defines inner, $B_{i}$, and outer, $B_{o}$, border cost functions:

$$
B_{i}=w_{i} H\left(I_{y}\right) I_{y}+\left(1-w_{i}\right) I_{y y}
$$


and

$$
B_{o}=w_{o}\left(1-H\left(I_{y}\right)\right) I_{y}+\left(1-w_{o}\right) I_{y y} .
$$

where $H$ is the Heaviside step function, returning 1 for positive values and 0 for negative values, $w_{i}$ and $w_{o}$ are weights applied to balance the effects of the two derivatives. In the case of a rising edge (the airway inner border) in the polar image $I_{y y}$ is going to reach its maximum value slightly before $I_{y}$. Adjusting the weights therefore enables a slight adjustment of the maximum of the cost function, in relation to the edge. Similarly for a falling edge (the airway outer border), where $I_{y}$ is going to reach its minimum slightly before $I_{y y}$ reaches its maximum. By using this fact the cost function maximum and thereby the likely found position can be adjusted by choosing weights to compensate for effects such as the size overestimation caused by the imaging system's PSF.

$B_{i}$ and $B_{o}$ not only contain positive peaks at the wanted border positions, but also smaller peaks at the outer and inner border positions respectively. We corrected this by defining positive and negative parts of $I_{y}$ in the following way:

$$
P=I_{y} H\left(I_{y}\right)
$$

and

$$
N=I_{y}\left(1-H\left(I_{y}\right)\right),
$$

The first order derivatives of these (in the y-direction), $P_{y}$ and $N_{y}$ were then used to define the new cost functions, $C_{i}$ and $C_{o}$ :

$$
C_{i}=w_{i} P+\left(1-w_{i}\right) P_{y}
$$

and

$$
C_{o}=\left(1-w_{o}\right) N_{y}-w_{o} N
$$

Derivatives were found using central differences. An example of $C_{i}$ and $C_{o}$ is shown in Figure $1(\mathrm{c})$ and $1(\mathrm{~d})$. Figure 2 illustrates the effects of applying the algorithm with different values of the inner and outer border weight parameters.

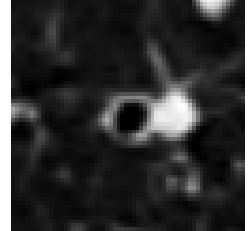

(a) Cross

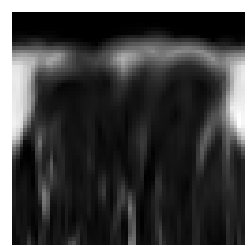

(b) Polar

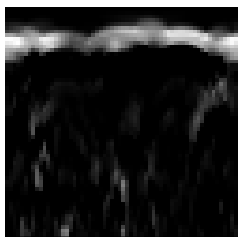

(c) Inner

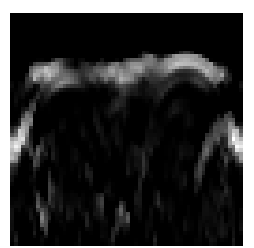

(d) Outer

Figure 1. Example of the transformation of the cross-section 1(a), to polar coordinates 1(b), which was then used as a basis for the calculation of the inner $1(\mathrm{c})$ and outer $1(\mathrm{~d})$ cost functions $C_{i}$ and $C_{o}$.

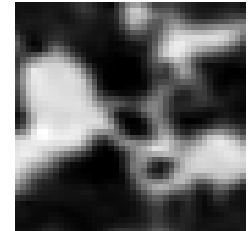

(a) Original

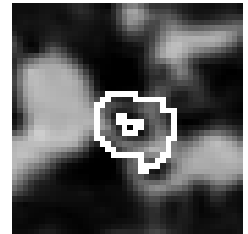

(b) Least

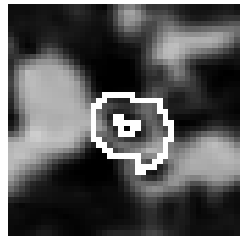

(c) Less

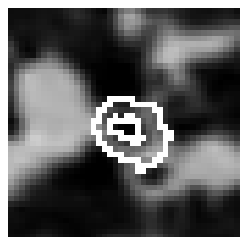

(d) Optimal

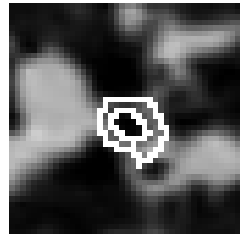

(e) More

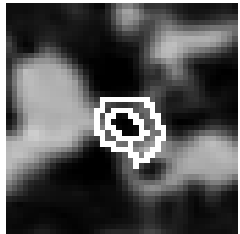

(f) Most

Figure 2. Results of changing the weight parameter for both the inner and outer border. The wall area decreases from left to right as the first order derivative becomes dominant. 2(d) shows the result of the optimal weight as determined by the training algorithm. 


\subsection{Coupled graph cut}

The Coupled Graph Cut (CGC) method presented in this paper is similar to Ref. 15 in that it combines the search for the inner and outer border into one graph cut using separation constraints and and it also uses smoothness constraints to keep the solutions continuous. However our graph construction technique uses soft constraints as opposed to the hard constraints of Ref. 15. Instead of having a hard border between feasible and infeasible solutions where a constraint becomes violated, our approach enables a smooth transition in which solutions simply become less likely as a function of how much they violate the constraint. An advantage of our method is that the constraints can be real valued since they are not a function of edge endpoints positions, enabling an infinite range of choices of how much to constrain the solutions.

First we describe how the inner and outer graph was constructed. Let $G_{i}$ and $G_{o}$ denote the inner and outer part of the graph, $C_{i}(x, y)$ and $C_{o}(x, y)$ with $x \in\{0,1, \ldots, X-1\}$ and $y \in\{0,1, \ldots, Y-1\}$ be the inner or outer cost function image with resolution $X \times Y$ constructed as described in Section 2.4. Then for each subscript $p \in\{i, o\}$ : Let $r_{p}$ be the intensity range of $C_{p}$ :

$$
r_{p}=\max \left(C_{p}(x, y)\right)-\min \left(C_{p}(x, y)\right)
$$

- There are $X \times(Y-1)+2$ vertices in $G_{p}$, for reference named $v_{p}(x, y)$, including a source, $s$ and a sink, $t$.

- $\forall x \in\{0,1, \ldots, X-1\}$ : there is a directed edge from $s$ to $v_{p}(x, 0)$ with capacity $r_{p}-C_{p}(x, 0)$ called $e_{p}(x, 0)$.

- $\forall x \in\{0,1, \ldots, X-1\}$ : there is a directed edge from $v_{p}(x, Y-2)$ to $t$ with capacity $r_{p}-C_{p}(x, Y-1)$ called $e_{p}(x, Y-1)$.

- $\forall x \in\{0,1, \ldots, X-1\}, \forall y \in\{1,2, \ldots, Y-2\}$ : there is a directed edge from $v_{p}(x, y-1)$ to $v_{p}(x, y)$ with capacity $r_{p}-C_{p}(x, y)$ called $e_{p}(x, y)$.

The smoothness constraint, $s_{p} \in\left[0, \infty\left[\right.\right.$, was implemented by adding angular edges with capacity $s_{p} r_{p}$ :

- $\forall x \in\{0,1, \ldots, X-2\}, \forall y \in\{1,2, \ldots, Y-2\}$ : there is a bi-directional edge from $v_{p}(x, y)$ to $v_{p}(x+1, y)$, with capacities $s_{p} r_{p}$

and similarly across the borders of the polar image:

- $\forall y \in\{1,2, \ldots, Y-2\}$ : there is a bi-directional edge from $v_{p}(0, y)$ to $v_{p}(X-1, y)$, with capacities $s_{p} r_{p}$.

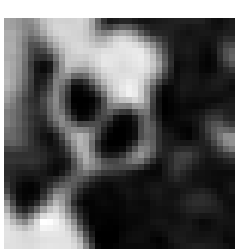

(a) Original

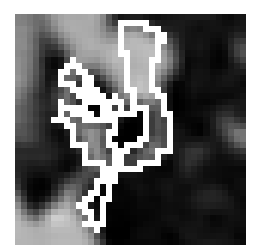

(b) Nothing

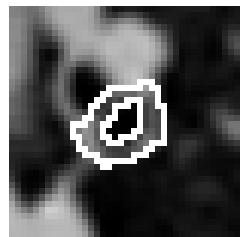

(c) Less

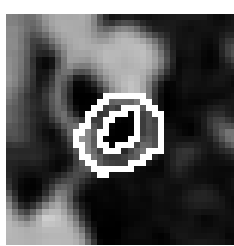

(d) Optimal

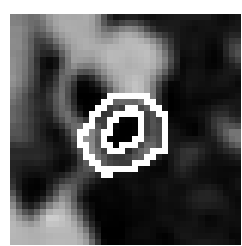

(e) More

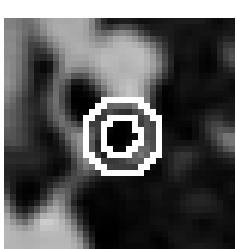

(f) A lot

Figure 3. Segmentations obtained using different values of the smoothness constraint for both the inner and outer border. The solution becomes increasingly round from left to right as the values are increased. 3(d) shows the optimal amount of smoothing as determined by the training algorithm.

Figure 3 shows results of running the algorithm with different values of the smoothness constraint.

Next we describe how the coupled surface graph, $G$, was constructed. Let the vertices of $G_{i}$ and $G_{o}$ be given by:

$$
\begin{gathered}
V_{i}=\left\{v_{i}(x, y) \mid x \in\{0,1, \ldots, X-1\}, y \in\{0,1, \ldots, Y-2\}\right\} \text { and } \\
V_{o}=\left\{v_{o}(x, y) \mid x \in\{0,1, \ldots, X-1\}, y \in\{0,1, \ldots, Y-2\}\right\}
\end{gathered}
$$

respectively and let the edges of $G_{i}$ and $G_{o}$ be denoted by $E_{i}$ and $E_{o}$ respectively, then the vertices, $V$, and edges $E$ of $G$, was constructed as: 
- $V=V_{i} \cup V_{o}$.

- $E=E_{i} \cup E_{o}$.

The separation constraint, $c$, is implemented by adding edges going from the inner to the outer part, as follows:

- $\forall x \in\{0,1, \ldots, X-1\}$ and $\forall y \in\{0,1, \ldots, Y-2\}$ : there is a bi-directional edge from $v_{i}(x, y)$ to $v_{o}(x, y)$ with capacities $c\left(r_{i}+r_{o}\right)$.

The larger the value of $c$ chosen, the closer the borders are likely to be. This is because the cut is a surface cutting both the inner and outer part of the graph. If the surface has different $y$-values in the inner and outer part, then it crosses these newly added edges and incurs the costs of these. Increasing the value of $c$ will, therefore, increase the likelihood that closer $y$-values are chosen. Figure 4 contains an illustration of this graph construction technique and Figure 5 shows results of running the algorithm with various values of the separation constraint parameter.

The algorithm described in Ref. 24 was used to calculate the minimum cut and the inner and outer borders were defined by the pixels $(x, y): e_{i}(x, y) \in$ cut and $(x, y): e_{o}(x, y) \in$ cut respectively.

Note that the above construction is essentially a way to make the likelihood that a given solution is chosen dependent on how close the wall width is to being 0 . It is possible to prioritize solutions of any width, $k$, by simply adding $k$ to the outer edge $y$-value endpoint index. We tried adding $k$ as a parameter in the training algorithm and this did lead to better segmentation results, however the trained values of $c$ increased a lot as well. This means that the increased accuracy probably came at a cost of decreased variability in the found wall widths and because the wall area percentage is one of the best measures of COPD we chose $k=0$. Further experiments are needed to determine whether a larger training set with more variation in wall width or perhaps adding two separation constraints in the form of an upper and a lower separation constraint can alleviate the problem.

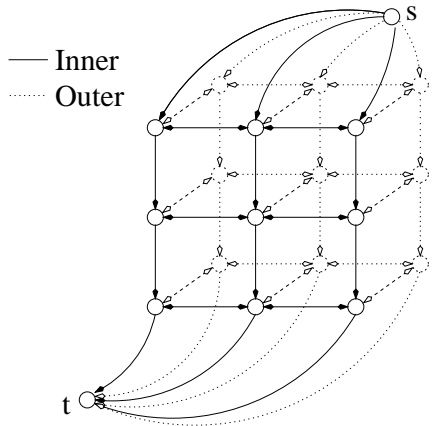

Figure 4. The coupled graph cuts construction method, $3 \times 4$ example. The wrap-around edges have been left out for clarity.

\subsection{Training}

The FWHM and CGC method described both contain parameters which can be adjusted for different results. Phantom data was not available as part of this study, so in order to judge how well given parameters work a training set of manually segmented cross-sections was constructed. The total number of misclassified voxels $E_{\text {voxels }}$ can be expressed as:

$$
E_{\text {voxels }}=\left|M_{i} \cup A_{i} \backslash M_{i} \cap A_{i}\right|+\left|M_{o} \cup A_{o} \backslash M_{o} \cap A_{o}\right|
$$

where $M_{i}$ and $M_{o}$ are the manual segmentations of the inner and outer areas respectively, $A_{i}$ and $A_{o}$ are the automatic segmentations, segmentations provided by the algorithms, of the inner and outer areas respectively 


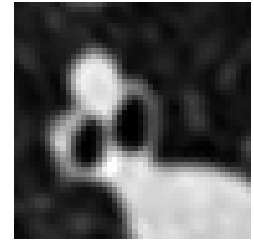

(a) Original

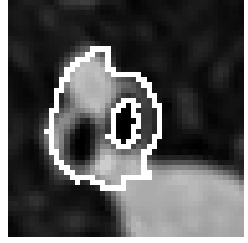

(b) Nothing

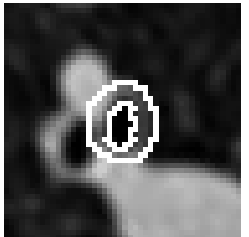

(c) Optimal

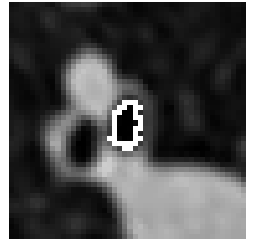

(d) Too high

Figure 5. Changing the separation constraint: 5(b) shows the result of having no separation constraint, 5(c) the optimal value as determined by the training algorithm and 5(d) a too high value causing borders to be on top of each other. The distance between the outer and inner borders generally becomes smaller as the separation constraint is increased, but the change is not continuous as the optimal solution is also determined by the cost function and smoothness constraint. The change in distance therefore only happens when the separation constraint makes a solution with smaller width the new optimal solution.

and $\cup, \cap$ and $\backslash$ are the standard set operations of union, intersection and set difference. The following error metric was used to judge a given segmentation during training:

$$
E=\frac{E_{\text {voxels }}}{S^{2}}
$$

Using averages of $E$ over the entire training set a binary search algorithm with random starting positions was used to search the parameter space and find the optimal combinations of parameter values.

\subsection{Measures}

Measurements were conducted on the already published measures: IA, WA \%, PI10, PWAt. Lacking phantom data, determining the best cutoff value for PI10 was not possible, so we used $6 \mathrm{~mm}$ as in Ref. 4. As investigated in Ref. 14 IA and WA \% are sensitive to the blurring effect of the PSF once the size of the measured structure becomes small enough. PWAt is expected to be more tolerant ${ }^{5}$ and captures density changes as well as size changes in structures close to the size of the PSF, which through the blurring effect changes the peak wall attenuation. Normalized Wall Intensity Sum (NWIS) is a new measure of mass changes within the wall, which because it is normalized by the total area of the airway, should capture changes in the mass of the airway wall whether they are caused by density changes or changes to the wall area at any size.

$$
\mathrm{NWIS}=\frac{\sum \mathrm{WA}(I+1000)}{\mathrm{WA}+\mathrm{IA}}
$$

where $I$ is the intensity function and WA the wall area. By adding 1000 to intensity values we effectively assume the area not included in the summation, IA, to have the density of air, which is -1000 Hounsfield Units.

\section{EXPERIMENTS AND RESULTS}

\subsection{Material}

Low-dose Computed Tomography (CT) images from the Danish Lung Cancer Screening Trial (DLCST) ${ }^{25}$ are used to tune segmentation parameters, evaluate and quantify. The data set consist of more than 2000 subjects scanned yearly (for 5 consecutive years), men and women, of age 50 to 70 years, smokers and former smokers with a history of at least 20 pack years. In order to be included each subject had to have a Forced Expiratory Volume in 1 second as a percentage of predicted value $\left(\mathrm{FEV}_{1}(\%\right.$ pred)) of at least 30 and weigh less than 130 $\mathrm{kg}$.

Lung function tests were performed annually following the recommendations of the European Respiratory Society. Used measures are: $\mathrm{FEV}_{1}$ calculated as a percentage of the predicted value $\left(\mathrm{FEV}_{1}(\%\right.$ pred $\left.)\right)$, ${ }^{25}$ which is based on age, sex and height and the ratio between $\mathrm{FEV}_{1}$ and Forced Vital Capacity $\left(\mathrm{FEV}_{1} / \mathrm{FVC}\right.$ ).

Subjects were scanned using a Multidetector CT (MDCT) scanner (16 rows Philips Mx 8000) at full inspiration with a low dose protocol $(120 \mathrm{kV}$ and $40 \mathrm{mAs}) .{ }^{25}$ Slice thickness was $1 \mathrm{~mm}$ with an approximate in-plane resolution of $0.78 \mathrm{~mm}$. 


\subsection{Results of training}

The training set was constructed of 188 cross-sections extracted from 15 different patients at random places in the airways.

The training algorithm was run until no further improvements in segmentation accuracy was made. These parameters were then accepted as optimal and used in the following experiments.

The optimal parameters for the cost function first and second order derivative weighting ended up being 0.701 for the inner border and 0.490 for the outer, showing that both derivatives were involved in computing the optimal cost function. The inner and outer smoothness constraints, separation constraint and the parameter determining the degree of first order derivative smoothing in the FWHM algorithm ended up being $0.168,0.215$, 0.015 and 1.01 respectively. Meaning all the algorithm parameters were used to optimally segment the training set.

\subsection{Comparison to manual segmentations}

An independent validation set was constructed similarly as the training set using 173 cross-sections. On this data, four different methods were compared: The coupled surface graph construction method with cost functions from Ref. 7 (BCF), the coupled surface graph construction method with the cost functions proposed in this paper (CGC), an independent surface graph as in Ref. 11 with the proposed cost functions (EG), and FWHM. The results are shown in Table 1.

Table 1. Manual data evaluation results. Area overestimation is determined as the average value of $100 \times|A \backslash M| /|M|$, similarly area underestimation is determined as the average value of $100 \times|M \backslash A| /|M|$. The average misclassified voxel count was determined using Equation 1.

\begin{tabular}{|r|r|r|r|r|r|}
\hline & FWHM & EG & BCF & CGC & Manual \\
\hline Average lumen area $\left(\mathrm{mm}^{2}\right)$ & 5.09 & 6.66 & 6.87 & 6.98 & 6.56 \\
Average airway area $\left(\mathrm{mm}^{2}\right)$ & 28.66 & 55.18 & 29.49 & 32.58 & 35.65 \\
Lumen area underestimation (\%) & 29.35 & 15.12 & 14.19 & 13.77 & \\
Lumen area overestimation (\%) & 7.82 & 20.07 & 20.44 & 21.45 & \\
Airway area underestimation (\%) & 30.25 & 8.42 & 22.66 & 15.12 & \\
Airway area overestimation (\%) & 11.06 & 63.64 & 7.06 & 8.71 & \\
Average misclassified voxel count & 16.93 & 27.81 & 12.84 & 10.62 & \\
\hline
\end{tabular}

Comparing EG and CGC is useful for evaluating the added benefits of the real valued smoothness constraint made possible by the proposed graph construction technique and the separation constraint parameter. The difference is large, with an overall improvement of $62 \%$ less misclassified voxels. The biggest improvement being in the degree of overestimation of the total airway area. This is in agreement with what was found in Ref. 15 single surface segmentation cannot accurately segment the outer border.

Comparing the data in the BCF and CGC columns is useful for assessing the improvements caused by the proposed cost functions. Although the difference is less than for the EG and CGC case, the total airway area is slightly less underestimated in the CGC case, leading to a better total error estimate.

The full width at half maximum method works well despite its simplicity. It has much less misclassified voxels than the EG method, but more than the CGC method.

\subsection{Performance of the algorithm as a COPD diagnostic tool}

The algorithm's ability to diagnose COPD was quantified using 144 healthy subjects, defined as having FEV ${ }_{1}$ $(\%$ pred $) \geq 80$ and $\mathrm{FEV}_{1} / \mathrm{FVC} \geq 0.7$ and $152 \mathrm{COPD}$ patients, defined as having $\mathrm{FEV}_{1}(\%$ pred $)<80$ and $\mathrm{FEV}_{1} / \mathrm{FVC}<0.7$, corresponding to GOLD Stage II and higher, ${ }^{2}$ all randomly chosen from the DLCST data. 
Table 2. Diagnosing COPD, Area Under receiver operating Characteristic (AUC).

\begin{tabular}{|c|c|c|c|c|c|}
\hline AUC & IA & WA $\%$ & PI10 & PWAt & NWIS \\
\hline CGC & 0.69 & 0.76 & 0.69 & 0.83 & 0.81 \\
FWHM & - & 0.66 & 0.65 & 0.83 & 0.81 \\
\hline
\end{tabular}

The data set is identical to the one used in another study on emphysema measures ${ }^{26}$ CGC was able to statistically separate the two groups $\left(p<10^{-7}\right)$ using all measures, compared to FWHM which could only separate the groups using WA\%, PI10, NWIS and PWAt $\left(p<10^{-5}\right)$. Both were performed using a Mann-Whitney U test. Figure 6 shows scatter plots of healthy and COPD subjects using CGC measures. Healthy subjects are scattered more towards the right in the IA plot and towards the left in the others. Indicating the relationship also confirmed by the correlation test described below. Interestingly the AUC values for the traditional emphysema measures $\mathrm{RA}_{950}, \mathrm{RA}_{890}$ and the developed kNN classifier all found in Ref. 26 are 0.59, 0.58 and 0.75 respectively, which are all lower than the CGC values of WA\%, NWIS and PWAt shown in Table 2. This indicates that airway measures of COPD, and of these especially the intensity based measures NWIS and PWAt, are just as important as emphysema based measures, for diagnosing COPD, if not more so.

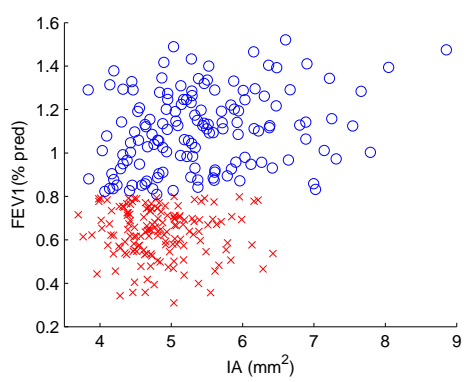

(a)

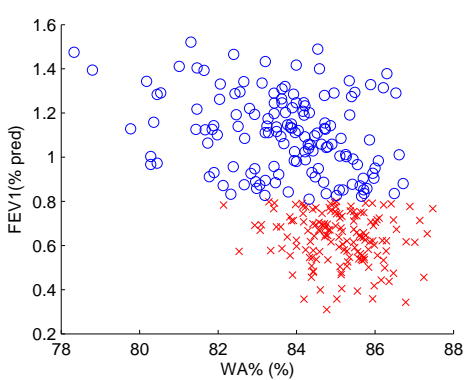

(b)

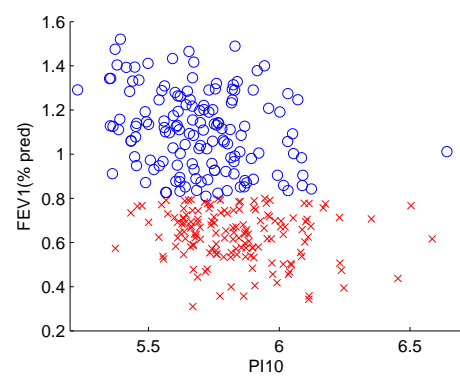

(c)

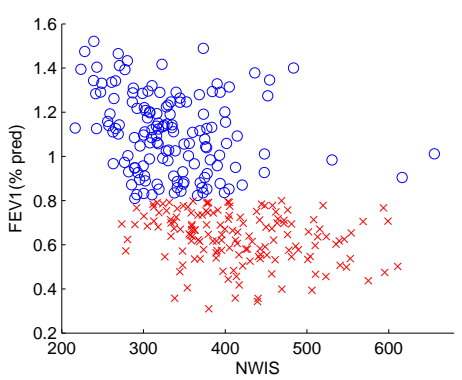

(d)

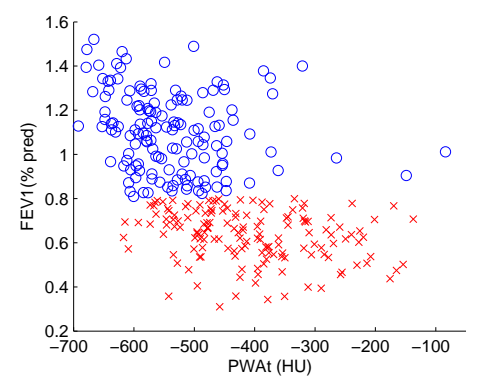

(e)

Figure 6. Scatter plots of COPD patients (red - $\mathrm{x}$ ) and healthy subjects (blue - o) measures IA (6(a)), WA\% (6(b)), PI10 (6(c)), NWIS (6(d)) and PWAt (6(e)) plotted against $\mathrm{FEV}_{1}$ (\% predicted).

Correlation with pulmonary function test values: $\mathrm{FEV}_{1}$ (\% predicted) and $\mathrm{FEV}_{1} / \mathrm{FVC}$, which are part of the standard COPD diagnosis method ${ }^{2}$ was examined using $250 \mathrm{CT}$ scans of just as many subjects randomly chosen from the DLCST data. The correlation coefficients and $p$-values are shown in Table 3. Correlation is both stronger and more significant in the CGC measurements, which suggests it outperforms FWHM as a measure of severity of COPD. Interestingly the intensity based measures: NWIS and PWAt show the largest correlations and are least affected by the change in algorithm, which might be an indication that they have larger segmentation error tolerance as well.

Reproducibility was quantified using 250 scan pairs taken with an interval of approximately one year also randomly chosen and all measures achieved significant correlation using Pearson product-moment correlation 
Table 3. Spearman's rank correlation coefficients and corresponding $p$-values as (r, $p$ )-pairs.

\begin{tabular}{|c|c|c|}
\hline CGC & $\mathrm{FEV}_{1}(\%$ pred $)$ & $\mathrm{FEV}_{1} / \mathrm{FVC}^{-4}$ \\
\hline IA & $0.26,2 \times 10^{-5}$ & $0.22,5 \times 10^{-4}$ \\
WA $\%$ & $-0.38,5 \times 10^{-10}$ & $-0.36,7 \times 10^{-9}$ \\
PI10 & $-0.31,4 \times 10^{-7}$ & $-0.29,3 \times 10^{-6}$ \\
PWAt & $-0.39,2 \times 10^{-10}$ & $-0.47,8 \times 10^{-15}$ \\
NWIS & $-0.40,7 \times 10^{-11}$ & $-0.43,2 \times 10^{-12}$ \\
\hline FWHM & $\mathrm{FEV}_{1}(\%$ pred $)$ & $\mathrm{FEV}_{1} / \mathrm{FVC}^{-1}$ \\
\hline IA & $0.04,5 \times 10^{-1}$ & $-0.09,2 \times 10^{-1}$ \\
WA $\%$ & $-0.26,3 \times 10^{-5}$ & $-0.18,4 \times 10^{-3}$ \\
PI10 & $-0.28,7 \times 10^{-6}$ & $-0.19,2 \times 10^{-3}$ \\
PWAt & $-0.38,3 \times 10^{-10}$ & $-0.47,4 \times 10^{-15}$ \\
NWIS & $-0.38,4 \times 10^{-10}$ & $-0.42,3 \times 10^{-12}$ \\
\hline
\end{tabular}

CGC: $\left(\mathrm{p}<10^{-43}\right)$ and FWHM: $\left(\mathrm{p}<10^{-15}\right)$ as seen in Table 4 . The CGC measures are more consistent.

Table 4. Repeat scan correlation, Pearson product-moment correlation coefficient.

\begin{tabular}{|c|c|c|c|c|c|}
\hline & IA & WA $\%$ & PI10 & PWAt & NWIS \\
\hline CGC & 0.63 & 0.74 & 0.58 & 0.83 & 0.83 \\
FWHM & 0.49 & 0.66 & 0.52 & 0.83 & 0.83 \\
\hline
\end{tabular}

Interestingly the intensity based measures are not only the most consistent measures, they are also least affected by the change in algorithm. As a comparison: $\mathrm{FEV}_{1}$ (\% pred) had a correlation coefficient of 0.93 , which is superior to the CT based measures.

\section{DISCUSSION AND CONCLUSION}

A new coupled surface graph cut method was presented, validated on manually segmented data and evaluated as a COPD diagnosis tool using pulmonary function tests. The proposed method finds more accurate segmentations than a similar algorithm using independent searches for the inner and outer border and the FWHM method when manually segmented images were used as a comparison, as shown in Table 1. The method enabled measurements of IA, WA\%, PI10, NWIS and PWAt which, with a few exceptions, correlated better with FEV 1 (\% pred) and $\mathrm{FEV}_{1} / \mathrm{FVC}$ than the same measures obtained using the FWHM segmentation algorithm. As observed in Table 4, measurements from the proposed method are equally or more reproducible than measurements obtained with FWHM. The measures were significantly different for patients with COPD stage II and higher compared to healthy subjects. Values of AUC were higher or equal to the FWHM algorithm.

Additionally a new intensity based measure of airway wall thickness was explored, which better discriminated subjects diagnosed with COPD stage II and higher from healthy subjects, correlated more with FEV 1 (\% pred) and $\mathrm{FEV}_{1} / \mathrm{FVC}$ and was more reproducible compared to traditional measures IA, PI10 and WA\%.

While further work is needed to match the reproducibility of $\mathrm{FEV}_{1}$ (\% pred) measurements, CT measures of airway wall thickness and emphysema such as the proposed method and Ref. 26, have the advantage of physically localizing the disease and enabling phenotyping of patients into disease groups. Studies such as Ref 27,28 have shown that CT based measurements may be more sensitive to changes in emphysema than lung function tests and future studies may reveal whether this is also the case for airway narrowing and wall thickening. This work shows that coupled surface graph cut methods such as the proposed method are well suited for such measurements. 


\section{ACKNOWLEDGMENTS}

This work is partly funded by the Danish Council for Strategic Research (NABIIT), the Netherlands Organisation for Scientific Research (NWO), and AstraZeneca, Lund, Sweden.

\section{REFERENCES}

[1] Hogg, J. C., Chu, F., Utokaparch, S., Woods, R., Elliot, W. M., Buzatu, L., Cherniack, R. M., Rogers, R. M., Sciurba, F. C., Coxson, H. O., and Paré, P. D., "The nature of small-airway obstruction in chronic obstructive pulmonary disease," The New England Journal of Medicine 350(26), 2645-2653 (2004).

[2] Rabe, K. F., Hurd, S., Anzueto, A., Barnes, P. J., Buist, S. A., Calverley, P., Fukuchi, Y., Jenkins, C., Rodriguez-Roisin, R., van Weel, C., and Zielinski, J., "Global strategy for the diagnosis, management, and prevention of chronic obstructive pulmonary disease: GOLD executive summary.," American Journal of Respitory and Critical Care Medicine 176, 532-555 (September 2007).

[3] Nakano, Y., Wong, J. C., de Jong, P. A., Buzatu, L., Nagao, T., Coxson, H. O., Elliott, W. M., Hogg, J. C., and Paré, P. D., "The prediction of small airway dimensions using computed tomography," American Journal of Respiratory and Critical Care Medicine 171, 142-146 (2005).

[4] Patel, B. D., Coxson, H. O., Pillai, S. G., Agusti, A. G. N., Calverley, P. M. A., Donner, C. F., Make, B. J., Müller, N. L., Rennard, S. I., Vestbo, J., Wouters, E. F. M., Hiorns, M. P., Nakano, Y., Camp, P. G., Fauerbach, P. V. N., Screaton, N. J., Campbell, E. J., Anderson, W. H., Paré, P. D., Levy, R. D., Lake, S. L., Silverman, E. K., Lomas, D. A., and on behalf of the International COPD Genetics Network, "Airway wall thickening and emphysema show independent familial aggregation in chronic obstructive pulmonary disease," American Journal of Respiratory and Critical Care Medicine 178, 500-505 (2008).

[5] Washko, G. R., Dransfield, M. T., Estépar, R. S. J., Diaz, A., Matsuoka, S., Yamashiro, T., Hatabu, H., Silverman, E. K., Bailey, W. C., and Reilly, J. J., "Airway wall attenuation: a biomarker of airway disease in subjects with COPD," Journal of Applied Physiology 107, 185-191 (April 2009).

[6] Estépar, R. S. J., Washko, G. G., Silverman, E. K., Reilly, J. J., Kikinis, R., and Westin, C.-F., "Accurate airway wall estimation using phase congruency," in [MICCAI (2)], 125-134 (2006).

[7] Kiraly, A. P., Odry, B. L., Naidich, D. P., and Novak, C. L., "Boundary-specific cost functions for quantitative airway analysis," in [MICCAI (1)], 784-791 (2007).

[8] Tschirren, J., Hoffman, E. A., McLennan, G., and Sonka, M., "Intrathoracic airway trees: segmentation and airway morphology analysis from low-dose CT scans," IEEE Trans. Med. Imaging 24(12), 1529-1539 (2005).

[9] Tschirren, J., Hoffman, E. A., McLennan, G., and Sonka, M., "Segmentation and quantitative analysis of intrathoracic airway trees from computed tomography images," Proceedings of the American Thoracic Society 2, 484-487 (2005).

[10] Weinheimer, O., Achenbach, T., Bletz, C., Düber, C., Kauczor, H.-U., and Heussel, C. P., "About objective 3-D analysis of airway geometry in computerized tomography," IEEE Trans. Med. Imaging 27(1), 64-74 (2008).

[11] Li, K., Wu, X., Chen, D. Z., and Sonka, M., "Efficient optimal surface detection: theory, implementation, and experimental validation," Medical Imaging 2004: Image Processing. Edited by Fitzpatrick, J. Michael; Sonka, Milan. Proceedings of the SPIE. 5370, 620-627 (2004).

[12] Lo, P., van Ginneken, B., Reinhardt, J. M., and de Bruijne, M., "Extraction of airways from CT (EXACT'09)," in [Proc. of Second International Workshop on Pulmonary Image Analysis], 175-189 (2009).

[13] Namkug, K., Beom, S. J., Sik, S. K., Jin, C. E., and Ho, K. S., "Semi-automatic measurement of the airway dimension by computed tomography using the full-width-half-maximum method: a study on the measurement accuracy according to the CT parameters and size of the airway.," Korean journal of radiology : official journal of the Korean Radiological Society 9, 226-235 (2008).

[14] Reinhardt, J. M., D'Souza, N. D., and Hoffman, E. A., "Accurate measurement of intrathoracic airways," IEEE Trans. Med. Imaging 16, 820-827 (December 1997).

[15] Li, K., Wu, X., Chen, D. Z., and Sonka, M., "Optimal surface segmentation in volumetric images-a graphtheoretic approach," IEEE Trans. Pattern Anal. Mach. Intell. 28(1), 119-134 (2006). 
[16] Greig, D. M., Porteous, B. T., and Seheult, A. H., "Exact maximum a posteriori estimation for binary images," Journal of the Royal Statistical Society. Series B (Methodological) 51(2), 271-279 (1989).

[17] Achenbach, T., Weinheimer, O., Biedermann, A., Schmitt, S., Freudenstein, D., Goutham, E., Kunz, R. P., Buhl, R., Dueber, C., and Heussel, C. P., "MDCT assessment of airway wall thickness in COPD patients using a new method: correlations with pulmonary function tests," European Radiology 18(12), 2731-2738 (2008).

[18] Berger, P., Perot, V., Desbarats, P., de Lara, J. M. T., Marthan, R., and Laurent, F., "Airway wall thickness in cigarette smokers: Quantitative thin-section CT assessment," Radiology 235, 1055-1064 (2005).

[19] Coxson, H. O., Quiney, B., Sin, D. D., Xing, L., McWilliams, A. M., Mayo, J. R., and Lam, S., "Airway wall thickness assessed using computed tomography and optical coherence tomography," American Journal of Respitory and Critical Care Medicine 177, 1201-1206 (2008).

[20] Lee, Y. K., Oh, Y.-M., Lee, J.-H., Kim, E. K., Lee, J. H., Kim, N., Seo, J. B., and Lee, S. D., "Quantitative assessment of emphysema, air trapping, and airway thickening on computed tomography," Lung 186, 277 (August 2008).

[21] Orlandi, I., Moroni, C., Camiciottoli, G., Bartolucci, M., Pistolesi, M., Villari, N., and Mascalchi, M., "Chronic obstructive pulmonary disease: Thin-section CT measurement of airway wall thickness and lung attenuation," Radiology 234, 604-610 (2005).

[22] Lo, P., Sporring, J., Ashraf, H., Pedersen, J. J. H., , and de Bruijne, M., "Vessel-guided airway segmentation based on voxel classification," The first International Workshop on Pulmonary Image Analysis 1, 113-122 (2008).

[23] Schlathölter, T., Lorenz, C., Carlsen, I. C., Renisch, S., and Deschamps, T., "Simultaneous segmentation and tree reconstruction of the airways for virtual bronchoscopy," in [SPIE International Symposium on Medical Imaging], (feb 2002).

[24] Boykov, Y. and Kolmogorov, V., "An experimental comparison of min-cut/max- flow algorithms for energy minimization in vision," Pattern Analysis and Machine Intelligence, IEEE Transactions on 26(9), 1124-1137 (2004).

[25] Pedersen, J. H., Ashraf, H., Dirksen, A., Bach, K., Hansen, H., Toennesen, P., Thorsen, H., Brodersen, J., Skov, B. G., Døssing, M., Mortensen, J., Richter, K., Clementsen, P., and Seersholm, N., "The danish randomized lung cancer CT screening trial-overall design and results of the prevalence round.," J Thorac Oncol 4, 608-614 (May 2009).

[26] Sørensen, L., Lo, P., Ashraf, H., Sporring, J., Nielsen, M., and de Bruijne, M., "Learning COPD sensitive filters in pulmonary CT.," in [MICCAI (1)], Yang, G.-Z., Hawkes, D. J., Rueckert, D., Noble, J. A., and 0002, C. J. T., eds., Lecture Notes in Computer Science 5762, 699-706, Springer (2009).

[27] Dirksen, A., Dijkman, J. H., Madsen, F., Stoel, B., Hutchison, D. C. S., Ulrik, C. S., Skovgaard, L. T., KokJensen, A., Rudolphus, A., Seersholm, N., Vrooman, H. A., Reiber, J. H. C., Hansen, N. C., Heckscher, T., Viskum, K., and Stolk, J., "A randomized clinical trial of $\alpha_{1}$-antitrypsin augmentation therapy," American Journal of Respiratory and Critical Care Medicine 160, 1468-1472 (1999).

[28] Dirksen, A., Piitulainen, E., Parr, D. G., Deng, C., Wencker, M., Shaker, S. B., and Stockley, R. A., "Exploring the role of CT densitometry: a randomised study of augmentation therapy in $\alpha_{1}$-antitrypsin deficiency," European Respiratory Journal 33, 1345-1353 (2009). 